\title{
Chapter 21. Anemia in CKD
}

(C) Japanese Society of Nephrology 2009

- CKD brings about renal anemia.

- Successful treatment of anemia may suppress decline of kidney function.

- The target level of renal anemia therapy is $\mathbf{H b}$ 10-12 g/dL.

- In management of anemia in CKD, evaluation of iron deficiency and appropriate iron supply are important.

\section{Renal anemia in CKD}

- Principally renal anemia is normocytic normochromic. Disorders of hematopoiesis lead to relative reduction in the number of reticulocytes.

- Renal anemia is caused mainly by impaired production of erythropoietin by the kidney and partly by uremic toxin.

- In renal anemia, erythropoietin concentration remains within normal or lower range, but its measurement is not essential for diagnosis.

- Renal anemia progresses so slowly that symptoms are usually not apparent.

- In CKD stages 3-5, the existence of anemia is periodically examined.

\section{Other causes of anemia in CKD}

- Anemia associated with CKD is most likely renal anemia, but differential diagnosis for other diseases is to be considered.
- In the presence of anemia in CKD stage 1-3, first of all, causative diseases other than renal anemia such as gastrointestinal bleeding are examined.

\section{Treatment of anemia protects the heart and kidney}

- Renal anemia is involved in progression of kidney dysfunction. Improvement of anemia by recombinant human erythropoietin agents (rHuEPO) was shown to suppress progression of kidney dysfunction (Fig. 21-1).

- Anemia is an exacerbating factor for heart failure, and treatment of anemia is beneficial for life expectancy.

- CVD is often associated with anemia, and treatment of anemia improves prognosis of CVD.

\section{The target level of anemia}

- The K/DOQI guidelines state that, in dialysis and nondialysis patients with $\mathrm{CKD}$ receiving $\mathrm{rHuEPO}$ therapy, the selected $\mathrm{Hb}$ target should generally be in the range $11.0-12.0 \mathrm{~g} / \mathrm{dL}$.

- In Japan, epoetin alfa or beta is administrated subcutaneously at initial dosage of 6,000 IU per injection per week until the target $\mathrm{Hb}$ level, followed by maintenance dosage of 6,000-12,000 IU per injection per 2 weeks.

- Upper limit of rHuEPO use approved by the health insurance system in Japan is 6,000-12,000 IU per 2 weeks, which sometimes fails to maintain $\mathrm{Hb}$ value above $11 \mathrm{~g} / \mathrm{dL}$. 


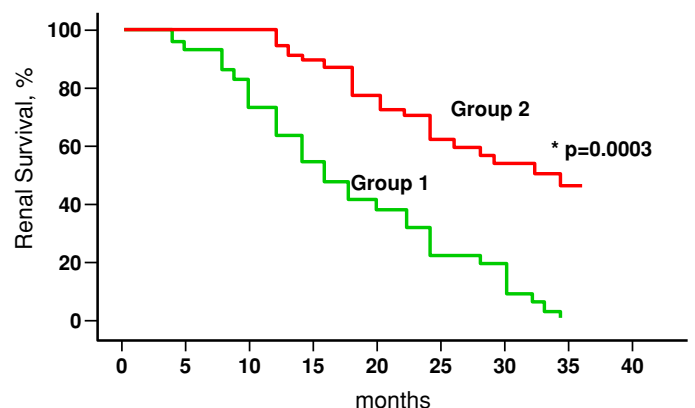

Group 1 : No erythropoietin $(\mathrm{n}=31), \mathrm{Hb}$ decreased from $9.3 \mathrm{~g} / \mathrm{dL}$ to $8.4 \mathrm{~g} / \mathrm{dL}$, Group 2 : Erythropoietin treated $(n=42), \mathrm{Hb}$ increased from $8.5 \mathrm{~g} / \mathrm{dL}$ to $11.8 \mathrm{~g} / \mathrm{dL}$.

Fig. 21-1 Effect of erythropoietin on renal survival. Quoted, with modification, from: Kuriyama S et al. Nephron, 1997;77:176-185

- The health insurance system in Japan requires that the target of anemia treatment with rHuEPO be around $10 \mathrm{~g} / \mathrm{dL}$ (or $30 \%$ in hematocrit level). Physicians are required to be careful not to raise $\mathrm{Hb}$ level above $12 \mathrm{~g} / \mathrm{dL}$ (or 36\% in hematocrit level).

- The Food and Drug Administration (FDA) called attention in March 2007 to the fact that higher chance of death and increased number of blood clots, strokes, heart failure, and heart attacks were reported in patients with CKD when rHuEPO were given to maintain hemoglobin levels of more than $12 \mathrm{~g} / \mathrm{dL}$.

- In Japan, there is not enough evidence for the target of anemia treatment in CKD, especially for its upper limit.

\section{Role sharing between nephrologists and primary care physicians in management of anemia}

- Start time and dosage of rHuEPO is determined through consultation with nephrologists, as CKD patients who require rHuEPO have severely reduced kidney function.
- Once a therapeutic strategy is decided, nephrologists and primary care physicians continue management in partnership with one another.

\section{Evaluation of iron deficiency in the treatment of anemia in CKD patients}

- Evaluation of iron deficit and proper iron supply is important in the treatment of anemia in CKD patients.

- Anemia in CKD patients may be improved by administration of iron supplements, even if iron deficiency is not apparent, as administration of rHuEPO causes relative iron deficiency.

- Excessive iron administration may causes hemosiderosis, so it is necessary during iron supply treatment to monitor ferrokinetic indices such as serum iron, total iron binding capacity, and ferritin. In particular, iron is administered with caution to CKD patients with chronic liver disease.

- The targets of anemia therapy with rHuEPO in CKD patients (from the K/DOQI guidelines) are:

\section{Serum ferritin $>100 \mathrm{ng} / \mathrm{mL}$}

\section{Transferrin saturation $($ TSAT) $>20 \%$}

TSAT $=$ Serum iron $(\mathrm{Fe}) /$ total iron binding capacity (TIBC)

- Iron can be administered either intravenously or orally. Intravenous route is required if iron deficiency is not sufficiently improved by oral administration or if oral administration is difficult due to gastrointestinal disorder or otherwise. Physicians are careful of allergic reaction or association with hemosiderosis. 